\title{
Two Dimensional MESFET Simulation of Transients and Steady State with Kinetic Based Hydrodynamical Models
}

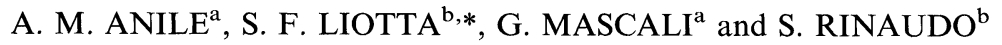 \\ ${ }^{a}$ Dipartimento di Matematica e Informatica, University of Catania, Viale A. Doria 6, 95125, Catania, Italy; \\ ${ }^{\mathrm{b}}$ ST Microelectronics, Stradale Primosole 50, 95121, Catania, Italy
}

\begin{abstract}
In this paper we compare the well-known and widely used hydrodynamical model of Blotekjaer-Baccarani-Worderman (BBW model) with a new hydrodynamical model recently proposed by Anile, Liotta and Mascali (ALM model). The benchmark utilized is a standard two-dimensional MESFET already employed in computational studies. Suitable numerical methods for hyperbolic systems of balance laws have been used.
\end{abstract}

Keywords: Boltzmann equation; Hydrodynamical models; MESFET simulations

\section{INTRODUCTION}

In modern electron devices, because of the presence of very high and rapidly varying electric fields, phenomena occur which cannot be described by means of the standard drift-diffusion (DD) models. For example, impact ionization and heat generation in the bulk material. Therefore generalizations of the DD equations have been sought which incorporate more dynamical variables, particularly the carrier energy. This has lead to the so-called energy-transport and hydrodynamical models.

One of the earlier and more widely known hydrodynamical models was put forward by Blotekjaer and subsequently investigated by
Baccarani and Wordeman and by other authors (BBW model) [1,2]. This model is implemented in simulation codes currently used in microelectronics industry. It consists of a set of balance equations for carrier density, momentum and energy obtained from the Boltzmann transport equation in the parabolic band approximation, closed by an assumed Fourier law as constitutive equation for the heat flux. The production terms for momentum and energy are assumed to be of the relaxation type and the relaxation times are obtained by phenomenological arguments. Since these assumptions are either ad hoc (like the Fourier law assumption) or at best phenomenological, in this paper we investigate a new hydrodynamical model recently proposed by Anile, Liotta and

* Corresponding author. 
Mascali (ALM model) [3], in which the closure of the equations is obtained in a systematic and rational way. In fact it is based on an asymptotic solution for high fields of the Boltzmann transport equation (BTE) for semiconductors recently found by Liotta and Majorana [4] and therefore it is not necessary to assume phenomenological closures or ad hoc expressions for the productions terms. All the parameters appearing in the model are explicitly calculated for a simple parabolic band silicon model in terms of the acoustic and optical phonon interactions constants. Extensions to more realistic band structures are under way and will be presented elsewhere.

From the mathematical viewpoint the resulting model is strictly hyperbolic. Therefore numerical methods suitable for hyperbolic systems of conservation laws have been used, in particular the well known Tadmor scheme for the convective step [5], completed with a suitable splitting strategy [6].

The benchmark used here is a standard MESFET model, already employed in computational studies [7].

\section{KINETIC BASED HYDRODYNAMICAL MODELS}

We treat the case of unipolar devices in which the current is essentially due to electrons. The semiclassical kinetic description [8] of the electron transport in semiconductors is based on the Boltzmann transport equation coupled to the Poisson equation for the electric potential ${ }^{1}$

$$
\begin{aligned}
& \frac{\partial f}{\partial t}+v_{i} \frac{\partial f}{\partial x_{i}}-\frac{q E_{i}}{\hbar} \frac{\partial f}{\partial k_{i}}=Q(f) \\
& \nabla_{\vec{x}} \cdot\left(\varepsilon \nabla_{\vec{x}} \Phi\right)=-q\left(N_{D}(\vec{x})-N_{A}(\vec{x})-n(\vec{x})\right)
\end{aligned}
$$

$f(\vec{x}, t, \vec{k})$ is the one-particle electron distribution function, with $\vec{k}$ the electron wave vector belonging to the first Briliouin zone $B, \vec{E}=-\nabla_{\vec{x}} \Phi$ the electric field, $\Phi$ the electric potential, $\hbar$ the reduced Planck constant, $\varepsilon$ material permittivity, $q$ the absolute value of the electron charge, $N_{D}$ and $N_{A}$ the concentrations of donor and acceptor impurities respectively, $n$ the electron density and $\vec{v}=1 / \hbar \nabla_{\vec{k}} \varepsilon(\vec{k})$ the electron group velocity which depends on the electron energy, $\varepsilon(\vec{k})$, in the conduction band (we assume only one conduction band for simplicity).

We will use the parabolic band approximation for which $\varepsilon(\vec{k})=\hbar^{2} k^{2} / 2 m^{*}, B=\Re^{3}, m^{*}$ being the electron effective mass.

$Q$ is the collision operator which in the nondegenerate case has the form [8],

$$
Q(f)=\int d \vec{k}^{\prime}\left[w\left(\vec{k}, \vec{k}^{\prime}\right) f\left(\vec{k}^{\prime}\right)-w\left(\vec{k}^{\prime}, \vec{k}\right) f(\vec{k})\right]
$$

$w\left(\vec{k}, \vec{k}^{\prime}\right)$ representing the electron scattering rate from a state with momentum $\vec{k}^{\prime}$ to one with momentum $\vec{k}$ (the $(\vec{x}, t)$ dependence of $f$ is not explicitly written).

Recently, in the case of bulk silicon, in homogeneous and stationary conditions, Liotta and Majorana (L.-M.) [4] found an asymptotic solution of the BTE, which is valid for electron energies, $\varepsilon(\vec{k})$, much greater than $\hbar \omega_{o p}$, energy of the optical phonon.

This analytic solution has been compared to the numerical one obtained under the same conditions [4] and the agreement between them has turned out to be very good even for low electron energies, at least in the case of sufficiently high electric fields. We use a macroscopic description of the devices based on the moments of the distribution function which correspond to the following kinetic quantities: $1, \vec{v}, \varepsilon$, that is

$$
n=\int_{\Re^{3}} f d \vec{k}, \quad \vec{u}=\frac{1}{n} \int_{\Re^{3}} \vec{v} f d \vec{k}, \quad W=\frac{1}{n} \int_{\Re^{3}} \varepsilon f d \vec{k}
$$

which are, respectively, the number density, the mean velocity and the mean energy of the electrons

\footnotetext{
${ }^{1}$ The Einstein convention is used throughout the paper.
} 
involved in the conduction current. The equations which describe the time evolution of these macroscopic fields are

$$
\begin{gathered}
\frac{\partial n}{\partial t}+\frac{\partial n u^{i}}{\partial x^{i}}=C_{n} \\
\frac{\partial n u^{j}}{\partial t}+\frac{\partial n K_{B} T^{i j}}{\partial x^{i}}+q E^{j} \frac{n}{m^{*}}=C_{w^{j}} \\
\frac{\partial n W}{\partial t}+\frac{\partial n S^{i}}{\partial x^{i}}+q E_{i} n u^{i}=C_{W}
\end{gathered}
$$

where the fluxes

$$
n K_{B} T^{i j}=\int_{\Re^{3}} v^{i} v^{j} f d \vec{k} \quad n S^{i}=\int_{\Re^{3}} \varepsilon v^{i} f d \vec{k}
$$

and the production terms

$$
\begin{aligned}
C_{n} & =\int_{\Re^{3}} Q d \vec{k} \quad C_{w^{j}}=\int_{\Re^{3}} v^{j} Q d \vec{k} \\
C_{W} & =\int_{\Re^{3}} \varepsilon Q d \vec{k}
\end{aligned}
$$

are extra-variables which are to be determined as functions of the fundamental variables $n, \vec{u}$ and $W$.

This is achieved, as said, by assuming that the electron distribution function depends on $n, \vec{u}$ and $W$ in the following manner

$$
\begin{aligned}
f= & \left(\frac{3 \hbar^{2}}{4 \pi m^{*} W}\right)^{3 / 2} n e^{-3 \varepsilon / 2 W} \\
& +\frac{27 \hbar^{3}}{32 \pi \sqrt{2 m^{*}}} \frac{n}{W^{2}} \varepsilon^{-1 / 2} e^{-3 \varepsilon / 2 W} u^{i} v_{i}
\end{aligned}
$$

which follows directly from the L.-M. distribution function $[3,4,9]$. Substituting it in the kinetic expressions of the fluxes (4), we obtain

$$
K_{B} T^{i j}=\frac{2}{3} \frac{W}{m^{*}} \delta^{i j} \quad S^{i}=\frac{4}{3} W u^{i} .
$$

As regards collisions, we will take into consideration the interaction between electrons and non-polar optical phonons and that between electrons and acoustical phonons, the latter in its elastic approximation which is valid when the thermal energy is much greater than that of the phonon involved in the scattering. Then the transition rate of the collision operator reads [10]

$$
\begin{aligned}
w\left(\vec{k}, \vec{k}^{\prime}\right)= & \mathcal{K}_{o p}\left[n_{o p} \delta\left(\varepsilon-\varepsilon^{\prime}-\hbar \omega_{o p}\right)\right. \\
& \left.+\left(n_{o p}+1\right) \delta\left(\varepsilon-\varepsilon^{\prime}+\hbar \omega_{o p}\right)\right] \\
& +\mathcal{K}_{a c} \delta\left(\varepsilon-\varepsilon^{\prime}\right)
\end{aligned}
$$

where $\mathcal{K}_{o p}$, and $\mathcal{K}_{a c}$ are respectively the nonpolar optical and acoustical scattering kernel coefficients, constant at a first approximation, and $n_{o p}$ is the thermal equilibrium optical phonon number.

The results relative to the production terms $\operatorname{are}^{2}$

$$
\begin{aligned}
& C_{n}=0 \quad C_{u^{i}}=-\frac{9 \pi \sqrt{2}}{\hbar^{3}} m^{* 3 / 2} \mathcal{K}_{o p} \\
&\left\{\alpha_{1}(W) n_{o p}+\alpha_{2}(W)\left(n_{o p}+1\right)\right. \\
&\left.+\alpha_{3}(W) \mathcal{K}\right\} \frac{n u^{i}}{W^{2}} \\
& C_{W}=\frac{12 m^{*} \sqrt{3 \pi m^{*}}}{\hbar^{3}} \hbar \omega_{o p} \mathcal{K}_{o p} \\
& \times\left\{n_{o p} \zeta_{1}(W)-\left(n_{o p}+1\right) \zeta_{2}(W)\right\} \frac{n}{W^{3 / 2}}
\end{aligned}
$$

with

$$
\begin{aligned}
\mathcal{K} & =\frac{\mathcal{K}_{a c}}{\mathcal{K}_{o p}} \\
\alpha_{1}(W) & =e^{3 \hbar \omega_{o p} / 2 W}\left[\frac{2}{3} W^{2} \frac{d \alpha_{0}(W)}{d W}-\hbar \omega_{o p} \alpha_{0}(W)\right] \\
\alpha_{2}(W) & =\frac{\sqrt{2 \pi}}{3 \sqrt{3}} W^{3 / 2} e^{-3 \hbar \omega_{o p} / 2 W}\left(W+\hbar \omega_{o p}\right) \\
\alpha_{3}(W) & =\frac{3 \sqrt{\pi}}{4}\left(\frac{2 W}{3}\right)^{5 / 2}
\end{aligned}
$$

${ }^{2} C_{n}=0$ means that the collision operator conserves the number of electrons. 
where

$$
\begin{aligned}
\alpha_{0}(W)= & \frac{\sqrt{2}}{2}\left(\frac{2 W}{3}\right)^{3 / 2}\left[1-\operatorname{erf}\left(\sqrt{\frac{3 \hbar \omega}{2 W}}\right)\right] \\
& +\frac{2 \sqrt{\hbar \omega}}{3} W e^{-3 \hbar \omega_{o p} / 2 W}
\end{aligned}
$$

erf is the error function, while

$$
\begin{aligned}
& \zeta_{1}(W)=\frac{1}{3} \hbar \omega_{o p} W e^{3 \hbar \omega_{o p} / 4 W} K_{1}\left(\frac{3 \hbar \omega_{o p}}{4 W}\right) \\
& \zeta_{2}(W)=e^{-3 \hbar \omega_{o p} / 2 W} \zeta_{1}(W)
\end{aligned}
$$

with $k_{1}(x)$ the second kind modified Bessel function of index 1 and argument $x$.

In [3] is shown that the system of balance laws (3) is strictly hyperbolic, and this permits us to use standard numerical methods developed for this kind of equations. In particular for the numerical integration of the system (3) we have used the two-dimensional extension of the Tadmor scheme [5] for the convective part completed with a splitting strategy to treat the scattering terms [6]. This method has the advantage that it is not necessary to know the eigenvalue structure of the hyperbolic system under consideration.

The numerical results obtained by numerically solving Eq. (3) in a suitable physical situation will be compared, in the next section, with those obtained by means of the standard BBW model, whose equations $[2,7]$, read

$$
\begin{aligned}
\frac{\partial n}{\partial t} & +\frac{\partial n u^{i}}{\partial x^{i}}=0 \\
\frac{\partial n u^{i}}{\partial t} & +\frac{\partial\left(n u^{i} u^{j}+n K_{B} T / m^{*} \delta_{i j}\right)}{\partial x^{j}} \\
& +q E^{i} \frac{n}{m^{*}}=-\frac{n u^{i}}{\tau_{p}} \\
\frac{\partial n W}{\partial t} & +\frac{\partial\left[\left(1 / 2 n m^{*} u^{2}+5 / 2 n K_{B} T\right) u^{i}-\kappa \nabla T\right]}{\partial x^{i}} \\
& +q E_{i} n u^{i}=-n \frac{W-W_{0}}{\tau_{w}} .
\end{aligned}
$$

Here $W$ is the electron total energy density $W=$ $\left(n m^{*} u^{2} / 2\right)+\left(3 n K_{B} T / 2\right)$, with $W_{0}=\left(3 n K_{B} T_{L} / 2\right)$ the equilibrium energy density ( $T_{L}$ lattice temperature) and $\tau_{p}, \tau_{q}$ phenomenological parameters to be interpreted as momentum and energy relaxation times (for details see ([7])). We want only to recall that in the BBW model the heat flux $\vec{h}$ is assumed to obey Fourier's law $\vec{h}=-\kappa \nabla T$.

\section{NUMERICAL SIMULATION RESULTS AND CONCLUSIONS}

Here we present some numerical simulations for a two-dimensional MESFET unipolar device of size $0.6 \times 0.2 \mu \mathrm{m}^{2}$ The source and the drain occupy $0.1 \mu \mathrm{m}$ respectively at the upper left and the upper right. The gate occupies $0.2 \mu \mathrm{m}$ at the upper middle. The doping is $n_{d}=3 \times 10^{17} \mathrm{~cm}^{-3}$ in the $n^{+}$regions and $n_{d}=1 \times 10^{17} \mathrm{~cm}^{-3}$ elsewhere, with abrupt junctions. A uniform grid of $72 \times 24$ points is used. We apply, at the source and drain, a voltage bias $V_{\text {bias }}=0.5 \mathrm{~V}$. The gate is a schottky contact, with a negative voltage bias $V_{\text {gate }}=$ $-0.8 \mathrm{~V}$. The lattice temperature is taken to be $T_{L}=300 \mathrm{~K}$. More details on this device can be found in [7].

As regards numerical simulation results, in Figure 1 we compare the electron densities obtained by using BBW and ALM models, and in Figure 2 electron temperatures. In Figures 3 and 4 densities, temperatures, horizontal and vertical velocities are compared, with cut at $y=0.183 \mu \mathrm{m}$. In Figure 5 we show the 2-D potential for ALM and compare potentials at the same cut. These figures are obtained at a time of 5 picosec, when a steady-state is reached. In Figure 6 we compare electron densities and temperatures after a time of 1 picosec. As is possible to see, the agreement during this transient state is good, but when the steady state is reached, the electron temperature obtained by using the ALM model is much higher than that in the BBW case. This behaviour is due to the asymptotic closure. Instead, the agreement between densities and horizontal velocities is good.

Some works about the extension of the ALM model in the case of a non-parabolic band of Kane 

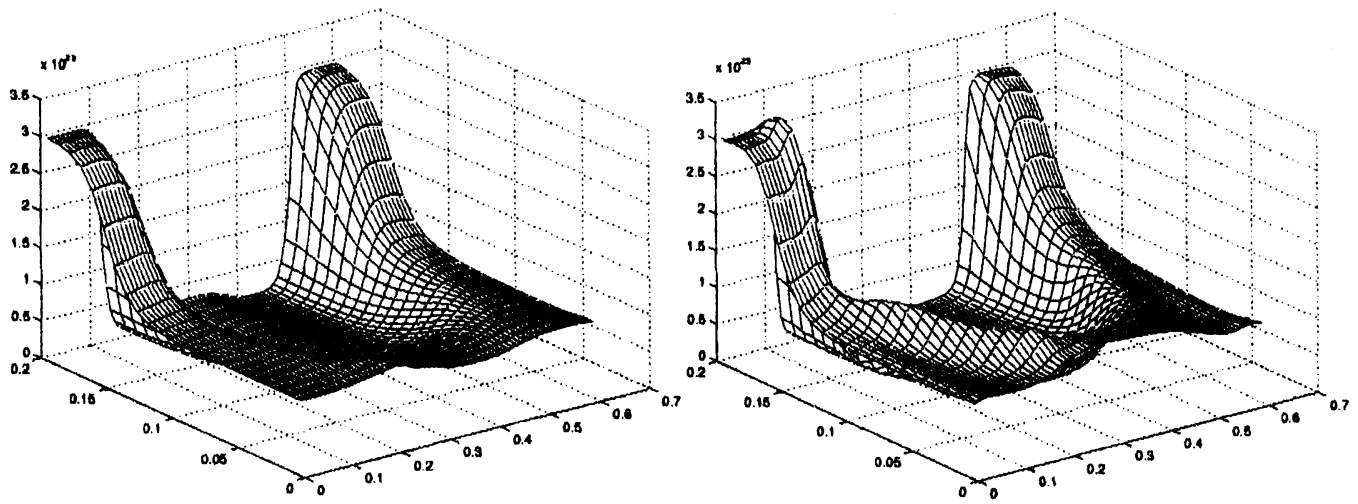

FIGURE 1 2-D MESFET. Electron density $\left(\mathrm{m}^{-3}\right)$ versus horizontal and vertical length $(\mu \mathrm{m})$. Left: BBW model, Right: ALM model.
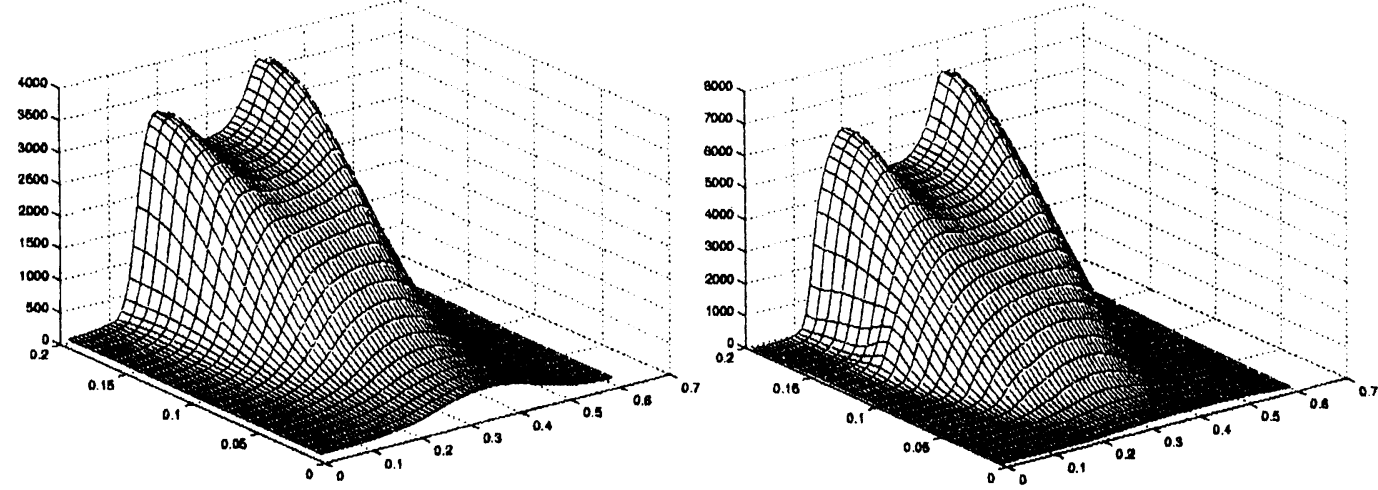

FIGURE 2 2-D MESFET. Electron temperature $(\mathrm{K})$ versus horizontal and vertical length $(\mu \mathrm{m})$. Left: BBW model, Right: ALM model.
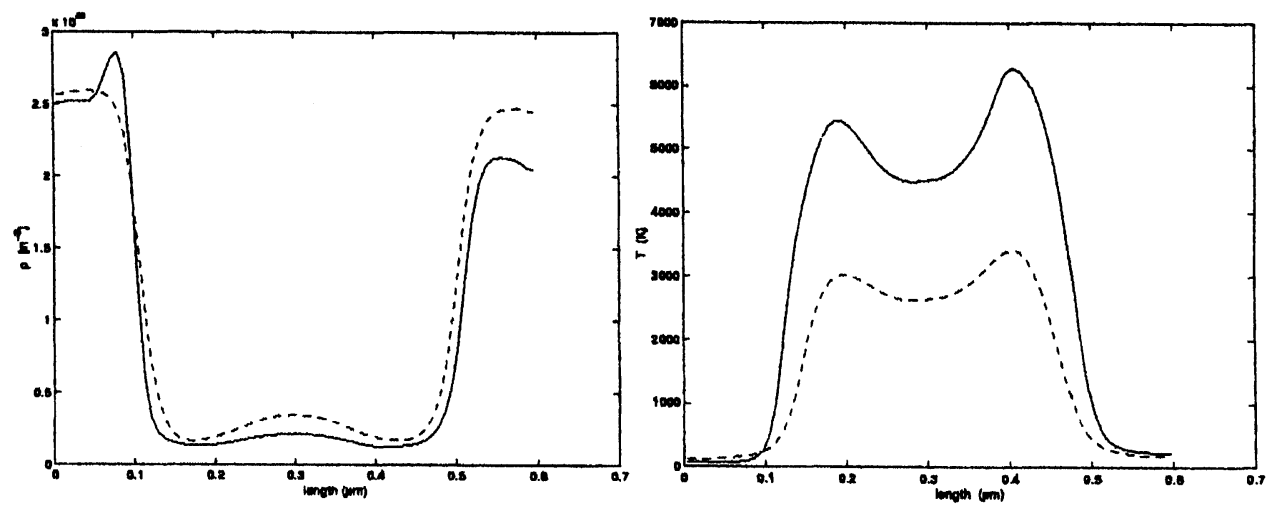

FIGURE 3 Comparison between BBW (dashed line) and ALM (continuous line) models. Left: density vs. length, Right: temperature vs. length. Cut at $y=0.183 \mu \mathrm{m}$. 

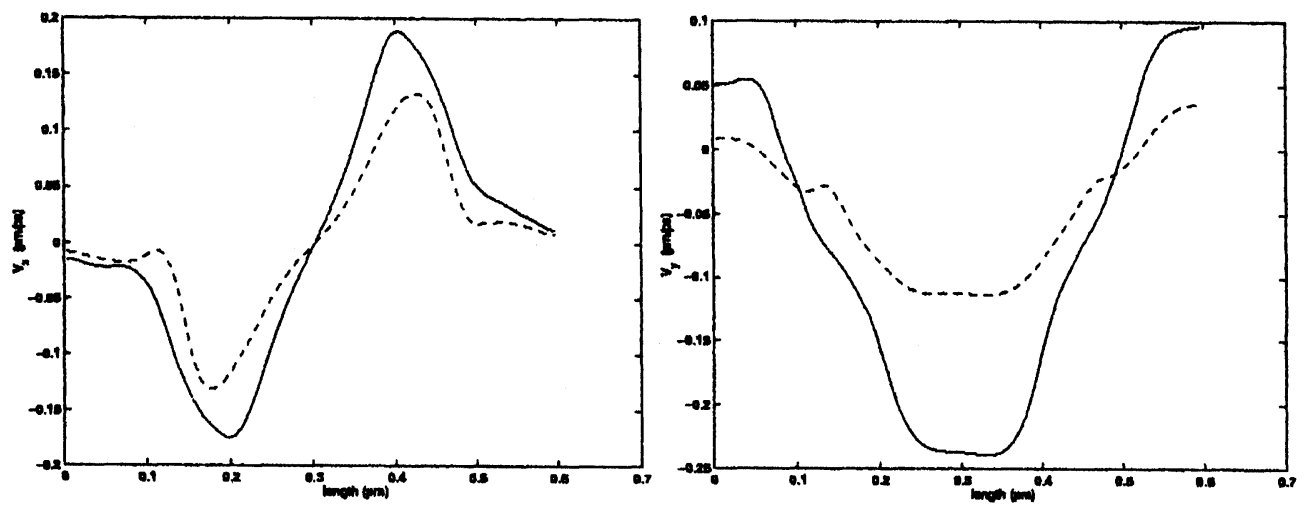

FIGURE 4 Comparison between BBW (dashed line) and ALM (continuous line) models. Left: orizontal velocity vs. length, Right: vertical velocity $v s$. length. Cut at $y=0.183 \mu \mathrm{m}$.
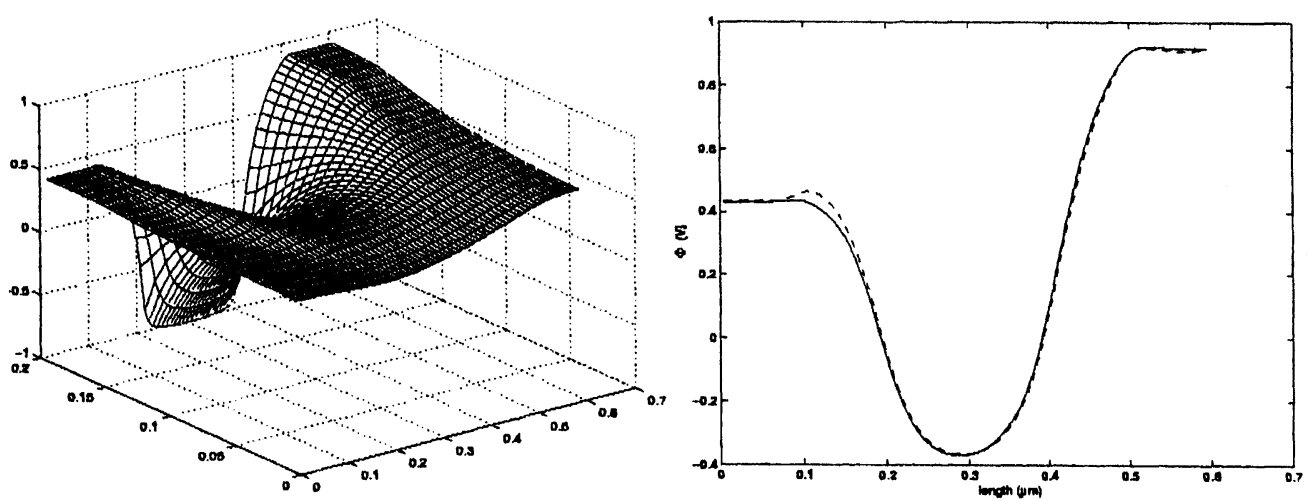

FIGURE 5 Left: 2-D potential for ALM model (Volts). Right: potential vs. length, comparison between BBW (dashed line) and ALM (continuous line). Cut at $y=0.183 \mu \mathrm{m}$.
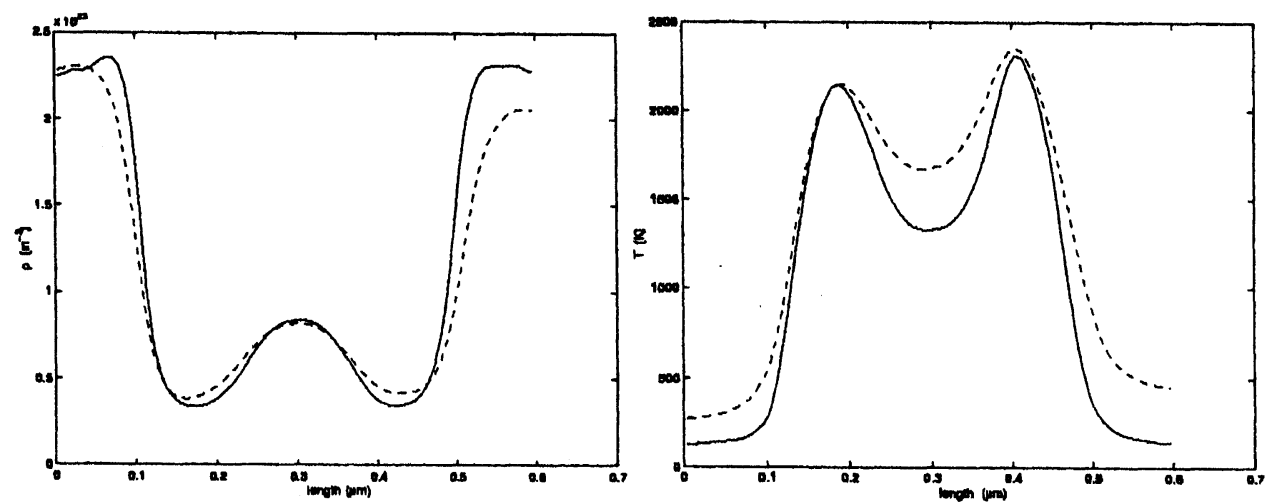

FIGURE 6 Comparison between BBW (dashed line) and ALM (continuous line) models. Left: density vs. length, Right: temperature $v s$. length. Time $=1$ picosec (transient). Cut at $y=0.183 \mu \mathrm{m}$. 
type, by using a new asymptotic solution recently found [11], are in progress. A better description of the hot electrons is expected.

\section{References}

[1] Blotekjaer (1970). Transport equations for electrons in two-valley semiconductors, IEEE Trans. Elec. Dev., 17, $38-47$.

[2] Baccarani, G. and Wordeman, M. R. (1985). An investigation of steady-state velocity overshoot in silicon, Solid-state Elec., 28(4), 407-416.

[3] Anile, A. M., Liotta, S. F. and Mascali, G., High field fluid dynamical models for the transport of charge carriers in semiconductors, submitted.

[4] Liotta, S. F. and Majorana, A., A novel approach to spherical harmonics expansion for electron transport in semiconductors, Preprint Univ. of Catania, available at this URL: http://arXiv.org/abs/cond-mat/ 0003456.
[5] Jiang, G. S. and Tadmor, E., Nonoscillatory central schemes for multidimensional hyperbolic conservation laws, SIAM J. Sci., Comput., 19(6), 1892-1917.

[6] Liotta, S. F., Romano, V. and Russo, G. (1999). Central Schemes for Systems of Balance Laws, International Series of Numerical Mathematics, Vol. 130, Birkhäuser Verlag Basel/Switzerland, pp. 651-660.

[7] Jerome, J. W. and Chi-Wang Shu, Semiconductors part II, the IMA volumes in Mathematics and its Applications, Coughran, N. M. Jr., Cole, J., Lloyd, P. and White, J. K. Editors, pp. 185-207.

[8] Markowich, A., Ringhofer, C. A. and Schmeiser, C. (1990). Semiconductor Equations, Springer-Verlag, Wein.

[9] Anile, A. M. and Mascali, G., Theoretical foundations for tail electron hydrodynamical models in semiconductors, to be published on Appl. Math. Lett.

[10] Jacoboni, C. and Lugli, P. (1989). The Monte Carlo method for semiconductor device simulation, New York, SpringerVerlag.

[11] Liotta, S. F., An asymptotic solution for the SHE equations describing the charge transport in semiconductors, Preprint Univ. of Catania, available at this URL: http://arXiv.org/abs/cond-mat/0003470. 

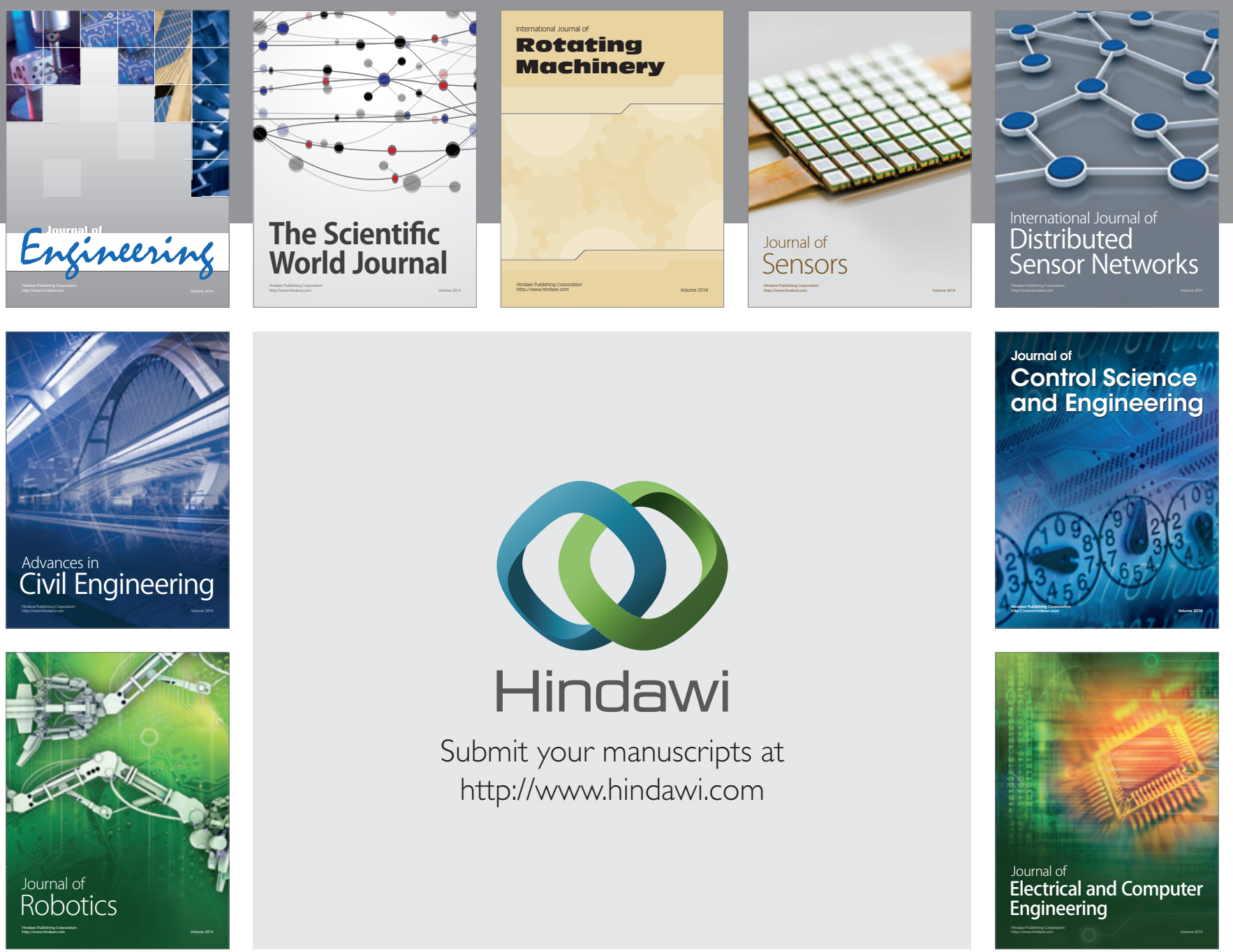

Submit your manuscripts at

http://www.hindawi.com
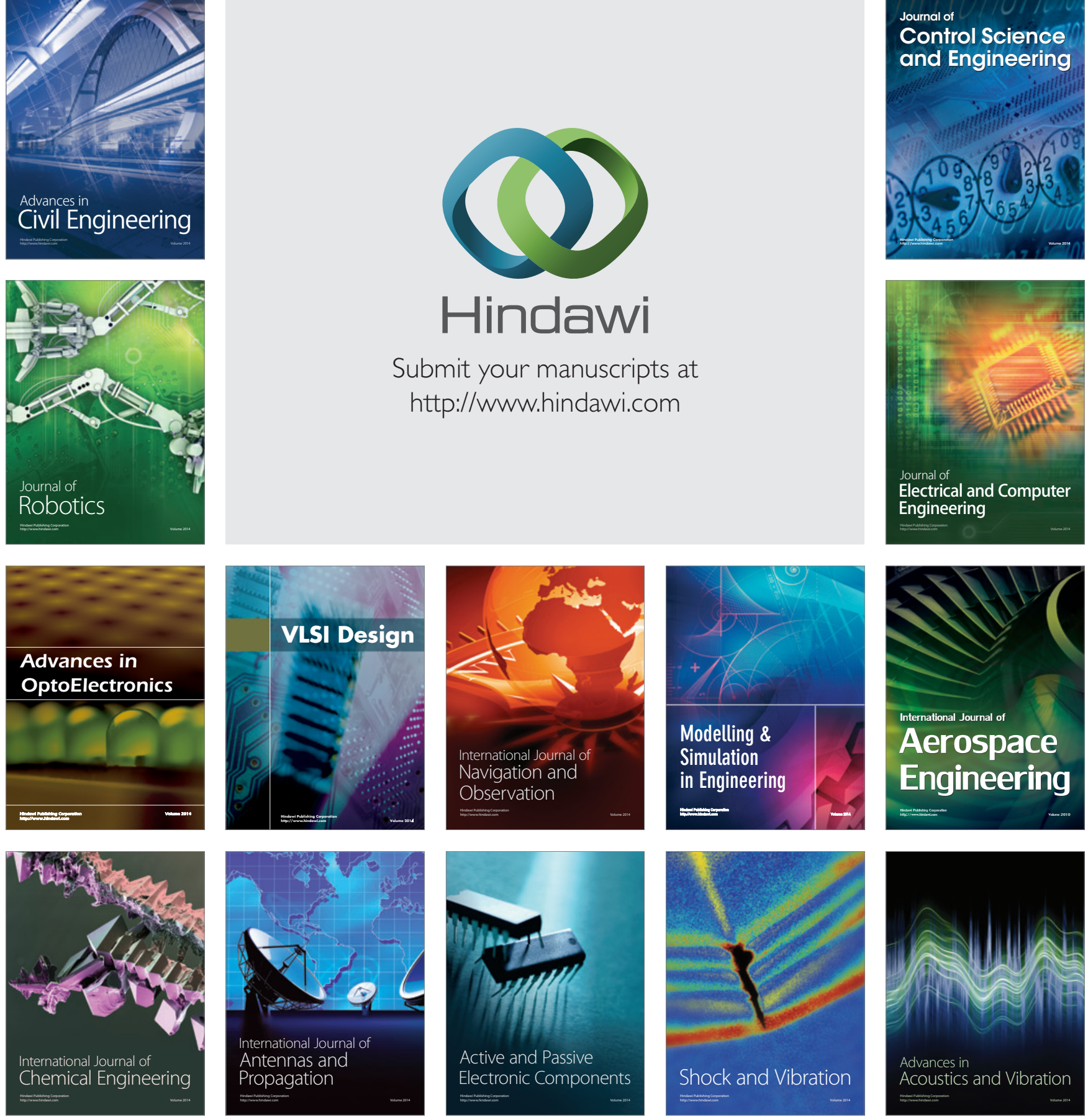